\title{
Determination of Drag From Three-Dimensional Viscous and Inviscid Flowfield Computations
}

\author{
David L. Hunt* \\ Aircraft Research Association \\ Bedford, UK \\ Russell M. Cummings $\dagger$ \\ California Polytechnic State University \\ San Luis Obispo, CA, USA \\ Michael B. Giles \\ Oxford University Computing Laboratory \\ Oxford, UK
}

\begin{abstract}
A momentum balance approach is used to extract the drag from flowfield computations for wings and wing/bodies in subsonic/transonic flight. The drag is decomposed into vorticity, entropy, and enthalpy components which can be related to the established engineering concepts of induced drag, wave and profile drag, and engine power and efficiency. This decomposition of the drag is useful in formulating techniques for accurately evaluating drag using computational fluid dynamics calculations or experimental data. A formulation for reducing the size of the region of the crossflow plane required for calculating the drag is developed using cut-off parameters for viscosity and entropy. This improves the accuracy of the calculations and decreases the computation time required to obtain the drag results. The improved method is applied to a variety of wings, including the M6, W4, and M165 wings, Lockheed Wing A, a NACA 0016 wing, and an Elliptic wing. The accuracy of the resulting drag calculations is related to various computational aspects, including grid type (structured or unstructured), grid density, flow regime (subsonic or transonic), boundary conditions, and the level of the governing equations (Euler or Navier-Stokes). The results show that drag prediction to within engineering accuracy is possible using computational fluid dynamics, and that numerical drag optimization of complex aircraft configurations is possible.
\end{abstract}

* Project Supervisor, CFD Research Group, email: dhunt@ara.co.uk. Member AIAA.

† Professor, Aeronautical Engineering Department, email: rcumming@calpoly.edu. Associate Fellow, AIAA.

$\$$ Rolls-Royce Reader in CFD, email: giles@comlab.ox.ac.uk. Member AIAA.

Copyright $(\mathcal{C} 1997$ by D.L. Hunt, R.M. Cummings, and M.B. Giles. Published by the American Institute of Aeronautics and Astronautics, Inc. with permission.

\section{Introduction}

As computational fluid dynamics (CFD) has matured over recent years, it has become a goal of CFD researchers to be able to predict aerodynamic drag from numerical simulations. Early attempts at accomplishing this were usually met with frustration, as most approaches involved integrating the pressure and skin friction over the surface of the body in order to calculate forces (the computational equivalent of force measurements in wind tunnels). Surface integration has met with difficulties, however, due to the need to approximate the curved surfaces of the body with flat facets. Calculation of the pressure and skin friction at the surface is also difficult at times. This has led various researchers to look at the experimental wake integral methods of Betz [1], Maull and Bearman [13], Maskell [11], Wu et al [22], and Brune and Bogataj [2], and to attempt to apply them to CFD computations. A good survey of drag computations methods was recently prepared by Takahashi [16] and may be useful to future researchers in understanding the uses and limitations of the experimental approaches. Computational methods involving wake integration have been shown to be reasonably accurate at predicting profile and vortex drag, as shown by van Dam and Nikfetrat [20], Chatterjee and Janus [4], and Van Der Vooren and Sloof [21]. An equivalent lifting-line approach by Mathias et al [12] has also been shown to be able to accurately compute induced drag.

A reformulation of the momentum balance equations for lift and drag has shown that near-field calculations could be performed which were as accurate as the traditional far-field analysis methods [6]. These improved integral relations need to be validated and applied to a variety of cases, including both Euler and Navier-Stokes computations, in order to determine the effect of the numerical approach on the accuracy of the drag extraction. Various contributing factors, such as the type of grid (structured or unstructured), the grid density in the wake region, the flow regime (subsonic or transonic), 
boundary conditions, outer boundary distances from the body, and the level of the governing equations (Euler or Navier-Stokes) need to be evaluated for their influence on the accuracy of the drag estimation. Once methods are developed which will make accurate drag calculations possible, then these procedures can be applied to design optimization algorithms which can be used to reduce the total drag of full configurations (including wings, bodies, and engine nacelles), including power effects. It will then be possible to optimally design an aircraft for overall reductions in total drag.

\section{Review of Theory}

By using a momentum balance approach, a system of integrals have been developed which reduce the task of force computation to the integration of various flow parameters in a crossflow plane downstream of a body [6]. The lift of the body can be related to the streamwise vorticity in the far-field as

$$
L=\rho_{\infty} U_{\infty} \iint y \zeta d y d z
$$

and the drag can be found to be made up of three components related to the entropy (viscous and wave drag), total enthalpy (engine thrust addition), and vorticity/stream function (induced drag)

$$
\begin{aligned}
& D \approx D_{1}+D_{2}+D_{3} \\
& D_{1}=p_{\infty} \iint \frac{s}{R} d y d z \\
& D_{2}=-\rho_{\infty} \iint \Delta H d y d z \\
& D_{3}=\frac{1}{2} \rho_{\infty} \iint \psi \zeta d y d z
\end{aligned}
$$

In an experiment or a computation, each of the integrals will be a weak function of the streamwise position of the crossflow plane on which they are evaluated. While moving downstream $D_{2}$ will approach a constant value of $-E / U_{\infty}$, where $E$ is the rate of energy addition in the engines. $D_{3}$ will decay very slowly to zero as the streamwise vorticity diffuses until the vorticity shed by one wing cancels the vorticity of the opposite sign shed by the other wing. In a CFD computation, because of numerical smoothing and coarse grids in the far-field, this will take place within the first 100 aircraft lengths; in reality it would take very much longer. As $D_{3}$ decreases

there is a corresponding increase in $D_{1}$ since the total drag remains a constant. In fact, the sum of the three components will be approximately constant well into the near-field of the aircraft. This is fortunate because both experimental measurements and CFD estimations of drag will usually have to be made in the near-field. Also, if a detailed breakdown of the sources of drag in a CFD calculation is required it is best to evaluate the three integrals in the near-field before numerical smoothing causes a shift from $D_{3}$ to $D_{1}$.

The integral relationships which were developed previously [6] used a Trefftz-plane concept and the following assumptions: 1) the crossflow plane must be aligned perpendicular to the freestream velocity vector, 2 ) the plane must include both wing-tips unless appropriate methods are used to compute the stream function/vorticity relationships in a half plane, and 3) the crossflow plane must be downstream of the aircraft. The third assumption creates practical limitations to the ability of the method to accurately predict the drag of a complex configuration (wing/fuselage/engine), in that the downstream crossflow plane may be far enough away from the lifting surfaces to have allowed a significant transfer of energy from $D_{3}$ to $D_{1}$. It may be useful, therefore, to place the crossflow plane relatively close to the trailing edge of the wing, which may cause the crossflow plane to include portions of the fuselage. This portion of the fuselage will affect the position and strength of the vorticity in the crossflow plane and lead to overpredicted values of lift, since lift is the integral of the product of vorticity and lateral position of the vorticity.

A possible method for correcting for the presence of the fuselage in a near-field crossflow plane is to replace the fuselage with a region of air with zero crossflow velocity. This would essentially create vorticity along the surface of the body, as in a wing vortex sheet. This vortex sheet could be used to correct the lift calculations for the presence of the body so that accurate predictions could be made even in the vicinity immediately behind the wing trailing edge. A correction of this sort will be shown in some of the following comparisons which include a wing/body configuration with a simulated sting extending downstream from the base of the fuselage. In these cases the crossflow plane has been placed behind the fuselage, but in the region which includes the sting. These computations show that it is possible to accurately correct for the presence of a body or sting when extracting lift from a crossflow plane.

\section{$\underline{\text { Basic Concepts }}$}

\section{Implementation}

For most CFD computations, especially for those using unstructured grids, there is no crossflow plane in the computational grid, and so the most natural approach for the evaluation of the crossflow drag integrals is to adopt techniques from flow visualization. A crossflow 'cutting plane' can be defined orthogonal to the freestream flow and at a fixed distance downstream of the aircraft. For an unstructured grid the grid nodes on the cutting plane are defined by the intersection of the plane and the edges of 
the three dimensional grid, and all flow variables can be defined at the new grid nodes by linear interpolation along the cut edges. The nodes of the cutting planes can be connected into triangles, based on the relationship of the cutting plane to the original cells. The full details for unstructured grids composed of tetrahedra, prisms, pyramids, and hexahedra are given in a paper by Giles and Haimes [8]. For calculations on single-block and multi-block structured grids, it is unlikely that there exists a suitable grid coordinate plane which is at a uniform streamwise distance downstream of the aircraft. One option is to use the same 'cutting plane' approach as was used for the unstructured grids, creating an unstructured triangular grid on the crossflow plane, with flow variable data interpolated along the cut edges of the structured grid.

The next issue is the interpretation of the values obtained from the drag integrals. Using CFD methods, it is possible to directly evaluate the aerodynamic forces on the aircraft using a numerical approximation of the surface integral. Almost all CFD methods are conservative, so if the surface force integration is performed in a manner consistent with the CFD discretisation of the cells with surface faces, then it is possible to sum over a very large number of computational cells surrounding the aircraft and deduce that the numerical surface integral is exactly equal to that which would be obtained by a numerical momentum integral applied on the enclosing control surface. In the far-field, numerical smoothing effects, like the real viscous effects, are very small. Therefore, the far-field asymptotic analysis remains valid, showing that the numerical force integral on the aircraft surface can be equated to the drag integrals on the crossflow plane.

\section{Need For Cutoffs}

A variety of practical issues arise when the momentum integrals for computing lift and drag are applied to either an experimental or computational set of data. Both sets of users want to reduce the size of the crossflow plane in order to reduce the time it takes to extract the forces: the wind tunnel experimenter wants to decrease the size of the crossflow survey to reduce tunnel occupancy time, and the CFD user wants to be able to compute forces in as close to real time as possible, especially when using the computations in conjunction with a numerical optimization scheme. In the wind tunnel, the wake surveys are restricted to a crossflow plane the size of the test section, but further reduction in probe survey sizes can be obtained by knowing that the wake region, including the trailing vortices from the wing-tips, lies in a fairly small region immediately behind the aircraft. It would be useful to be able to mimic this type of survey in the evaluation of drag using CFD results. The problem lies in determining the correct parameters to be used to determine when the crossflow plane is "large enough" to give accurate results.

Initial applications of the crossflow plane integration to CFD results showed that if the outer boundaries were too close to the body, a large contamination to the integration could take place. Crossflow plane cells at the outer boundary are usually quite large, and since the integrals essentially sum the difference between local flow variables and freestream values over a finite area, any small deviation in the flow variables from freestream conditions near the outer boundary can lead to large errors in the integration. Integrations which were performed in [6] showed that forces such as lift could be in error by as much as $50 \%$ if the outer boundary were included. This large error is due to a magnification of small errors by the multiplication of the vorticity by the lateral distance (see Eqn. 1). A simple reduction in the size of the crossflow plane showed that accurate calculations of the lift and drag could be obtained if the crossflow plane was as small as a one chord radius around the wing surface. Further reductions in the size of the crossflow plane showed a rapid decrease in the accuracy as regions of high vorticity and entropy changes would then be neglected in the integration. However, the precise size and shape of the required crossflow plane should be determined by the aircraft geometry and the resulting flowfield, which makes using a simple radius cutoff value for reducing the size of the crossflow plane problematic. There would be no a priori way to determine the right size of the crossflow plane for various geometries, i.e., a high aspect ratio wing at high angle of attack would require a larger radius crossflow plane than a small aspect ratio wing at low angles of attack. For this reason, a more suitable way to reduce the size of the crossflow plane would be to delete various cells from the integration based on the amount of vorticity and/or entropy in the cell, rather than on the distance of the cell from the wing.

\section{Cutoff Formulation (Elliptic Wing Case)}

In order to show that the lift and drag of an aircraft could be well predicted using reduced crossflow plane sizes, a simple test case was run. An elliptic planform wing with aspect ratio of seven $M_{\infty}=0.55$ and $\alpha=4^{\circ}$.was chosen for this study. Euler computations were performed using the SAUNA CFD system $[16,17]$. This wing has been computationally studied by van Dam and Nikfetrat [20], and computations were performed to match those cases. Bounding boxes were used to decrease the size of the crossflow plane; a bounding box of $N$ chords includes everything within a box outlined by $-N<Y<+N$ and $-N<Z<+N$. Figs. 1 and 2 show the effect of the size of the bounding box on the computation of lift and drag.

Figure 1 shows that the bounding box can be as small as three chords without effecting the prediction of lift, but that a bounding box which includes the outer boundary (fourteen chords) does not predict the lift accurately (as 
was explained in the previous section and reported in [6]). In addition, the lift is consistently predicted with crossflow planes which are as much as seven chords behind the wing (discrepancies in the results at crossflow plane conditions aft of seven chords are due to being in the proximity of the outflow boundary). The vortex drag (Fig. 2) is not affected by the size of the bounding box, but the values do decease as the crossflow plane is moved further aft of the wing. This is due to numerical dissipation converting crossflow kinetic energy into entropy $\left(D_{3}\right.$ to $\left.D_{1}\right)$.

These results show that the size of the crossflow plane can be reduced significantly and still result in consistent predictions of lift and drag. However, if the goal is to minimize the size of the crossflow plane in order to reduce computation time, then this method may not be the best choice since the size of the bounding box will not be consistent from configuration to configuration.

The inability of the simple radial cutoff to accurately predict the shape of the wake points to the need for some other type of cutoff parameter to determine which cells contain significant levels of vorticity and entropy. Since the goal is for the integration to include cells which contain the wake, the first level of cutoff was needed to eliminate cells which had less than a certain proportion of freestream reference levels of entropy and vorticity, given by

$$
\begin{aligned}
& s_{\infty}=\frac{\gamma}{2} R M_{\infty}^{2} \\
& \zeta_{\infty}=2 U_{\infty} / \sqrt{S_{\text {ref }} A R}
\end{aligned}
$$

where $M_{\infty}$ is the freestream Mach number, $\gamma$ is the ratio of specific heats, $R$ is the universal gas constant, $S_{\text {ref }}$ is the wing reference area, and $A R$ is the wing aspect ratio. The first entropy and vorticity cutoff parameters (Level 1), $C_{s_{1}}$ and $C_{\zeta_{1}}$, are defined as threshold levels proportional to the freestream values (Eqns. 6 and 7). The entropy and vorticity within a cell are included in the force integration if the cell can be shown to be directly connected to the wake and have entropy or vorticity levels such that

$$
\begin{aligned}
& s_{\text {cell }}>C_{s_{1}} s_{\infty} \\
& \zeta_{\text {cell }}>C_{\zeta_{1}} \zeta_{\infty}
\end{aligned}
$$

Since these cutoff levels are relative to the freestream values, they will be constant for all crossflow planes in the wake.

A second set of cutoff parameters (Level 2) for the entropy and vorticity, $C_{s_{2}}$ and $C_{\zeta_{2}}$, were defined relative to the maximum values in each crossflow plane, $s_{\max }$ and $\zeta_{\max }$. These parameters were found to be necessary since a global cutoff might not include smaller levels of entropy and vorticity in crossflow planes far downstream of the wing. The entropy and vorticity in the cells were included in the integration if

$$
\begin{aligned}
& s_{\text {cell }}>C_{s_{2}} s_{\max } \\
& \zeta_{\text {cell }}>C_{\zeta_{2}} \zeta_{\max }
\end{aligned}
$$

Values of the various cutoff parameters were determined from computations using the elliptic wing.

Figures 3 and 4 show the variation of lift and vortex drag coefficients for various values of $C_{\zeta_{1}}$. This figure shows that the lift integration can be consistently made with cutoff values between 0.001 and 0.1 , but that cutoff values either above or below these levels seriously degrade the accuracy of the prediction. The vortex drag is consistently predicted for cutoff values between 0 and 0.1 , with poor predictions occurring for cutoff values above 0.1 .

This points to the need for a combination of two levels of cutoff values for vorticity: one relative to the freestream (Level 1) and one relative to local conditions in the crossflow plane (Level 2). Figures 5 and 6 show the results with two vorticity cutoff values, a Level 1 value which varies as shown, and a constant Level 2 value of $C_{\zeta_{2}}=0.1$. These results show that the lift and vortex drag are consistently predicted for all combinations of cutoff values and at crossflow plane positions within six chords of the wing. This leads to choosing default values for the two vorticity cutoff parameters of $C_{\zeta_{1}}=0.01$ and $C_{\zeta_{2}}=0.1$. A similar determination of the entropy cutoff values (Fig. 7) shows that the entropy drag is consistently predicted for cutoff values of $C_{s_{1}}=0.003$ and $C_{s_{2}}=0.1$.

The final model for deciding which cells to use in the integration is to determine whether or not a cell has a level of entropy or vorticity above the threshold levels in the crossflow plane (Level 2). If the cell meets this criterion, and can be shown to be directly connected to other cells which form the wake, then it is used in the integration. Level 1 thresholds are then checked to see if other cells exist which have levels of entropy or vorticity which may be relatively large enough to be part of the wake, but the cell is only included if it also connects to other wake cells. In general, Level 1 thresholds are 
lower than Level 2 thresholds, and provide a means for fine tuning the size and shape of the wake.

Results for the elliptic wing with the newly determined cutoff values are presented in Fig. 8. These show the vorticity, entropy, and total drag coefficients as a function of streamwise position downstream of the wing (in chord lengths). When the default levels of cutoff values are applied to the total drag estimation various interesting, but not unexpected results take place. First of all, the total drag does not vary a great deal at various positions downstream of the wing (total drag in the wake should be invariant as was discussed previously in [6]). The vorticity drag decreases continuously the farther downstream the crossflow plane is placed, and the entropy drag is continuously increasing. This is the interchange of vorticity for entropy which is caused by numerical dissipation as the vortex convects downstream. It is because of this transfer of drag that the near-field is the best place for computation of drag from CFD results. This shows the value of the crossflow plane integral formulation, which was developed for use in the near field.

\section{Applications}

\section{M6 Wing}

The ONERA M6 Wing [15] was used as a basis for determining the effect of grid density and grid type on drag prediction using the entropy and vorticity cutoff methods. Both structured and unstructured grids were used with the following grid densities: 1) structured grid with 4,000 points (coarse grid), 27,000 points (medium grid), and 189,000 points (fine grid); 2) unstructured grid with 33,898 nodes and 185,239 cells (coarse mesh), 55,127 nodes and 314,518 cells (medium mesh), and with 97,533 nodes and 576,234 cells (fine mesh). Note that the grid densities between the two grid systems do not match since the computations were done with different Euler codes: the unstructured grid used the Cindy code [5] and the structured grid used the SAUNA code $[16,17]$. Both sets of computations were performed for $M_{\infty}=0.84$ and $\alpha=6.06^{\circ}$. The entropy and vorticity cutoffs were set to the default values which had been developed for the elliptic wing; $C_{\zeta_{1}}=0.01, C_{\zeta_{2}}=0.1, C_{s_{1}}=0.003$, and $C_{s_{2}}=0.1$.

Figures 9 and 10 show the results of the lift integration for the two grid types (structured and unstructured, respectively) as a function of distance downstream from the wing leading edge (in wing root chords). The results show that the lift is consistently predicted by the structured medium and fine grids, but not for the coarse grid. Likewise, the unstructured grids all show consistent results for crossflow plane positions withn seven or eight chords of the wing. Figures 11 and 12 show similar results for the entropy drag, and Figs. 13 and 14 show the vortex drag. These results show that the medium and fine structured and unstructured grids give consistent results, but that the structured and unstructured grids yield different levels of entropy drag (the vortex drag results are consistent between the two grid types). This variation could be due to different grid densities in the vicinity of the shock wave on the wing, which shows the need to cluster grids in this region in order to be able to accurately predict wave drag.

The total drag results for the structured and unstructured fine grids are compared in Figs. 15 and 16, respectively. Each figure includes the entropy, vorticity, and total drag. Both codes yield total drag results of approximately $C_{D}=0.06$, with consistent values for the vortex drag. While the total drag seems fairly constant for both grid types, there is an obvious exchange of entropy drag for viscous drag as the crossflow plane is moved further downstream. However, this trend stops after five chord lengths for the structured grid, while continuing for the unstructured grid.

\section{W4 Wing-Body}

The W4 configuration is a civil transport wing-body which is used to show the ability of the method to handle drag estimation for a geometry which includes the presence of a fuselage. This configuration has an aspect ratio of eight and was experimentally tested at $M_{\infty}=0.78$ and $\alpha=1.523^{\circ}$ by Fulker [7]; Euler computations were made to match these conditions using the SAUNA code $[16,17]$.

If the computational geometry includes a fuselage, or a simulated sting extending from the base of the fuselage, problems can arise in the computation of drag. The fuselage or sting pose a problem when applying the crossflow plane integration concept since the original integral formulation assumed that the crossflow plane was a Trefftz-plane. The sting creates a boundary surface within the plane which acts to force the trailing vortex away from the configuration centerline. This will adversely effect the prediction of lift, which is the integral of the product of the vorticity and the lateral location of the vorticity. When the vortex is forced further outboard the lateral position is increased, which causes an overprediction of lift. The corresponding calculation of drag components is not affected by the body since the drag integrals are not a function of the position of the vorticity. As mentioned previously, this problem can be rectified by assuming that the sting can be replaced by a region of zero crossflow velocity fluid. This fluid gradient creates the equivalent of a vortex sheet along the surface of the sting, the circulation of which can be determined and included in the lift calculations. This should reduce the computed lift, effectively correcting the lift value for the presence of the body in the crossflow plane. This 
approach would also allow for placing the crossflow plane immediately behind the trailing edge of the wing, so that detailed force information for the wing could be obtained in spite of the presence of the body.

The W4 wing-body geometry was run without the fuselage correction in order to determine the feasibility of predicting drag within the region of the fuselage. The geometry was first run to determine the level of the $C_{s_{1}}$ which would give consistent results; the value of $C_{s_{2}}$ was set to the default value of 0.1 , as was determined from the previous test cases. Figure 17 shows that the entropy drag is consistent for $C_{s_{1}}>0.0001$.

A study of the grid density in the wake of the wing was also conducted. Three grids were tested, each with a different number of axial planes aft of the wing: 13, 27 , and 27 planes. The entropy drag and vortex drag results for these three cases are presented in Figs. 18 and 19. The entropy drag results are fairly scattered for the grids which contain 13 and 27 planes, with a smoother variation of entropy drag for the grid with 37 wake planes. The vortex drag is well predicted by all three wake grid sizes, showing that the vortex drag (and therefore the lift) requires much lower grid density in the wake to give accurate and consistent results. The prediction of entropy drag in the wake, however, requires higher levels of grid density.

The resulting values of entropy, vortex, and total drag are shown in Fig. 20. These results use the grid with 37 planes in the wake and the default entropy and vorticity cutoff values of $C_{\zeta_{1}}=0.01, C_{\zeta_{2}}=0.1, C_{s_{1}}=0.003$, and $C_{s_{2}}=0.1$. The results show similar trends to the previous cases: an interchange of vortex and entropy drag, with a resulting total drag which is nearly constant throughout the wake. The corresponding lift for this case was predicted to be $C_{L}=0.75$, and is constant throughout the wake.

\section{M165 Canard-Wing}

The M165 configuration is a simple canard-wing which will show the effect of computing the forces on multiple lifting surfaces which are at different longitudinal positions in the configuration. The configuration has an aspect ratio of 2.1 and was wind-tunnel tested at $M_{\infty}=0.9$ and $\alpha=6^{\circ}$ by Stanniland [17]; Euler computations from the SAUNA code $[16,17]$ were made to match the experimental case. The default values for the cutoff parameters were used for this case.

The crossflow plane is positioned at various positions behind the trailing edge of the wing. Since the wing is behind the canard, the transfer of vorticity drag to entropy drag for the canard has already begun to take place at the position of the wing. In spite of this, the results in Fig. 21 show that the overall drag of the configuration is consistently predicted. As was seen in previous cases, the total drag is constant at $C_{D}=0.033$, with the majority of the total coming from vortex drag. The interchange between vortex drag and entropy drag is also apparent for this case.

\section{NACA 0016}

This is a simple rectangular wing model using an untwisted NACA 0016 airfoil section which was experimentally tested by Brune and Bogataj [2]. The test model had an aspect ratio of six with rounded wing tip fairings, and was tested at $M_{\mathrm{o}}=0.18, \alpha=8.22^{\circ}$, and $\operatorname{Re}=1.26 \times 10^{6}$. An incompressible Navier-Stokes CFD prediction of the flowfield around this wing was conducted by Mathias et al [12], including lift and drag estimates using both surface integration and an equivalent lifting line model (results are presented in Table 1). The computations utilized a single zone structured $\mathrm{C}-\mathrm{O}$ grid with three grid densities: coarse $(61 \times 61 \times 51)$, medium $(61 \times 61 \times 81)$, and fine $(81 \times 81 \times 81)$. Results for all three grid densities were previously presented [6], but the vorticity/entropy cutoff methods have now been applied to the fine grid.

Figure 22 shows the drag results for the wing as a function of distance downstream from the wing leading edge. Since this is the first viscous case where the cutoff parameters were applied it was nbecessary to see if the vorticity/entropy cutoffs used for the inviscid cases still yielded reasonable results. This wing does not have shock waves, but does have viscous effects included, so the general trends are somewhat different; in general, it will not be possible to determine the difference between wave drag and viscous drag within the entropy integral.

The total drag is overpredicted in the region immediately behind the wing, and continues to decrease at various positions downstream of the wing. It is not possible to determine if a general exchange of entropy drag for vortex drag takes place as the crossflow plane is moved further downstream, since the entropy drag already exists at fairly high levels due to the shed boundary layer behind the wing's trailing edge. The general trends hold farther than one chordlength downstream of the trailing edge, but the region close to the trailing edge exhibits a rise in vorticity drag where the boundary layer is shed into the wake. The computed lift-to-drag ratio at three chordlengths behind the wing is $L / D=14.9$, which underpredicts the experimental values of $L / D=17.6$ from a wake survey and $L / D=17.9$ from a balance measurement. The total drag is not constant as a function of distance from the crossflow plane, which may point to problems with artifical entropy which is created at the 
surface of the wing has been convected into the wake, which makes it very difficult to determine the actual drag. This type of problem will need to be investigated further.

\section{Lockheed Wing A}

The Lockheed Wing A has a planform which is representative of an advanced commercial transport wing and was extensively wind-tunnel tested by Hinson and Burdges [10]. The wing has an aspect ratio of eight, a taper ratio of 0.4 , a leading edge sweep of 27 degrees, and uses a $12 \%$ thick airfoil which is continuously twisted from the root to the tip. The flowfield about the wing was computed by Greenman et al [9] using the OVERFLOW compressible Navier-Stokes solver [3] and a single block structured grid. The computations were made at $M_{\infty}=0.82, \alpha=1.5^{\circ}$, and $\operatorname{Re}=6.0 \times 10^{6}$, which is representative of a cruise flight condition for a commercial transport.

Figure 23 shows the entropy, vorticity, and total drag for Wing A. These results show trends which are similar to previous results for the various inviscid cases, namely that the total drag is constant for as much as 30 chords downstream of the wing. The effects of the outflow boundary then begin to contaminate the crossflow plane data and cause inaccuracies in the prediction. The entropy drag is seen to increase throughout much of the wake, with a corresponding decrease in vortex drag. The total lift-to-drag ratio for the crossflow plane integration is $L / D=14.5$, which compares well with the experimental value of $L / D=13.4$. The lack of difficulties with the entropy drag for this case may point to different types of artificial viscosity having effects on the quality of the drag prediction.

\section{Conclusions}

A previously developed wake integral formualation for the prediction of lift and drag (both entropy drag and vortex drag) has been used on a variety of test cases. Methods for reducing the computational time required for integrating the wake crossflow planes have been developed so that the process can be used in conjunction with numerical optimization algorithms. Entropy and vorticity cutoff parameters have been developed relative to freestream reference levels and maximum levels in the crossflow plane. Cells in the crossflow plane which contain small proportions of these two levels of entropy and vorticity are not included in the integration. The methods have been applied to a variety of inviscid and viscous cases in order to determine the levels of the cutoff parameters for consistent prediction of lift and drag. Further applications of these concepts may lead to the accurate and efficient extraction of forces from CFD computations.

\section{Acknowledgments}

The authors wish to thank Karlin Roth, Roxana Greenman, and David Baker of NASA Ames Research Center, Donovan Mathias of Stanford University, and G. Shrinivas of the Oxford University Computing Laboratory for making available crossflow plane information for the Lockheed Wing A, NACA 0016, and unstructured M6 wing solutions, respectively. This work was partially funded by Rolls Royce plc and DRL.

\section{References}

[1] A. Betz, "Ein verfahren zur direkten ermittlung des profilwiderstandes," $Z F M$, Vol. 16, 1925, pp. 42-44.

[2] G.W. Brune and P.W. Bogataj, "Induced drag of a simple wing from wake measurements," SAE Technical Paper 901934, Oct. 1990.

[3] P.G. Buning, W.M. Chan, K.J. Renze, D.L. Sondak, I. Chiu, J.P. Slotnick, R.J. Gomez, D.C. Jespersen, S.E. Krist, and Y.M. Rizk, "OVERFLOW Manual Version 1.6bc," NASA Ames Research Center, Moffett Field, CA.

[4] A. Chatterjee and J.M. Janus, "On the use of a wakeintegral method for computational drag analysis," AIAA Paper 95-0535, Jan. 1995.

[5] P.I.Crumpton, "An efficient cell vertex method for unstructured tetrahedral grids," Numerical Methods for Fluid Dynamics V, Ed. K.W. Morton and M. J. Baines, Oxford: Clarendon Press, 1995, pp. 369-376.

[6] R.M. Cummings, M. Giles, and G. Shrinivas, "Analysis of the elements of drag in three-dimensional viscous and inviscid flows," AIAA Paper 96-2482, June 1996.

[7] J.L. Fulker, "A selection of experimental test cases for the validation of CFD codes-Case B3", AGARD AR 303, 1994.

[8] M.B. Giles and R. Haimes, "Advanced interactive visualization for CFD," Comput. Systems Engrg., Vol. 1, No. 1, 1990, pp. 51-62.

[9] R.M. Greenman, S. Cheung, and E.L. Tu, "Optimization: a tool to study the physical features of a transonic wing," AIAA Paper 96-2486, June 1996.

[10] B.L. Hinson and K.P. Burdges, "Acquisition and application of transonic wing and far-field test data for three-dimensional computational method evaluation," AFOSR-TR-80-0421, March 1980.

[11] E.C. Maskell, "Progress towards a method for the measurement of the components of the drag of a wing of 
finite span," RAE Tech Report 72232, 1973.

[12] D.L. Mathias, J.C. Ross, and R.M. Cummings, "Wake integration to predict wing span loading from a numerical simulation," J. Aircraft, Vol. 32, No. 5, Sep. 1995, pp. 1165-1167.

[13] D.J. Maull and P.W. Bearman, "The measurement of the drag of bluff bodies by the wake tranverse method," $J$. Roy. Aero. Soc., Vol. 68, Dec. 1964, p. 843.

[14] S.E. Rogers, "Numerical solution of the incompressible Navier-Stokes equations," NASA TM 102199, Nov. 1990.

[15] V. Schmitt and F. Charpin, "Pressure distribution on the ONERA-M6-Wing at transonic Mach numbers," AGARD AR-138, 1979.

[16] J.A. Shaw, A.J. Peace, J.M. Georgala, P.N. Childs, "Validation and evaluation of the advanced aeronautical CFD system SAUNA-a method developer's view," European Forum on Recent Development and Application in Aeronautical CFD, Bristol, U.K., 1993.

[17] J.A. Shaw, A.J. Peace, N.E. May, M.K. Pocock, "Verification of the CFD simulation system SAUNA for complex aircraft configurations," AIAA Paper 94-0393, Jan. 1994.

[18] D.R. Stanniland, "A selection of experimental test cases for the validation of CFD codes-Case D5", AGARD AR 303, 1994.

[19] T.T. Takahashi, "On the decomposition of drag from wake survey measurements," AIAA Paper 97-0717, Jan. 1997.

[20] C.P. van Dam and K. Nikfetrat, "Accurate prediction of drag using Euler methods," J. Aircraft, Vol 29, No. 3, 1992, pp. 516-519.

[21] J. Van Der Vooren and J.W. Sloof, "CFD-based drag prediction: state-of-the-art, theory, prospects," NLR Technical Report TP 90247, 1990; also "Lecture notes for the AIAA professional studies series course on drag prediction and measurement," 1990.

[22] J.C. Wu, J.E. Hackett, and D.E. Lilley, "A generalized wake-integral approach for drag determination in three-dimensional flows," AIAA Paper 79-0279, Jan. 1979.

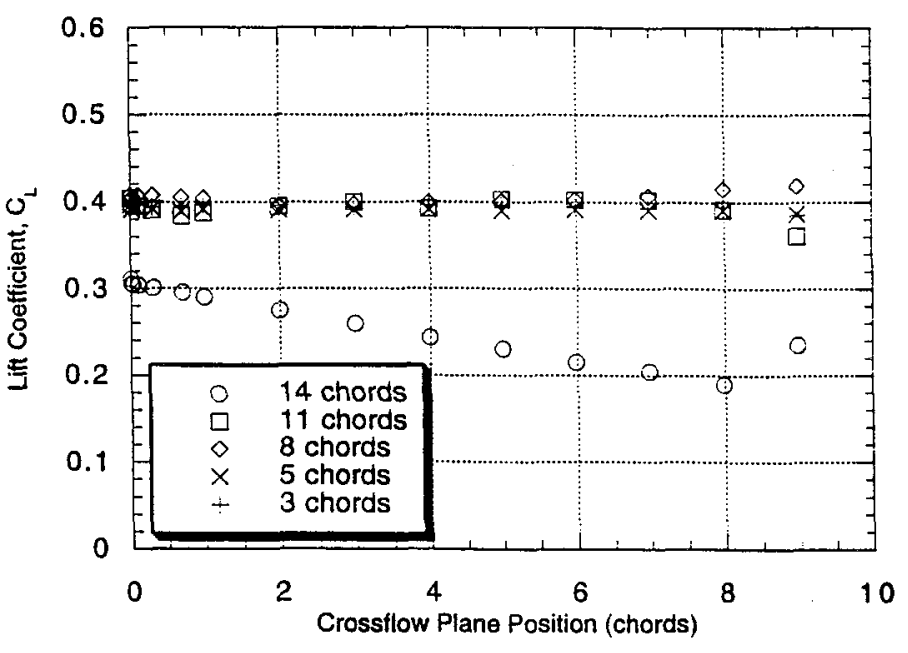

Fig. 1 Elliptic Wing-Effect of Bounding Box Size on Lift Coefficient.

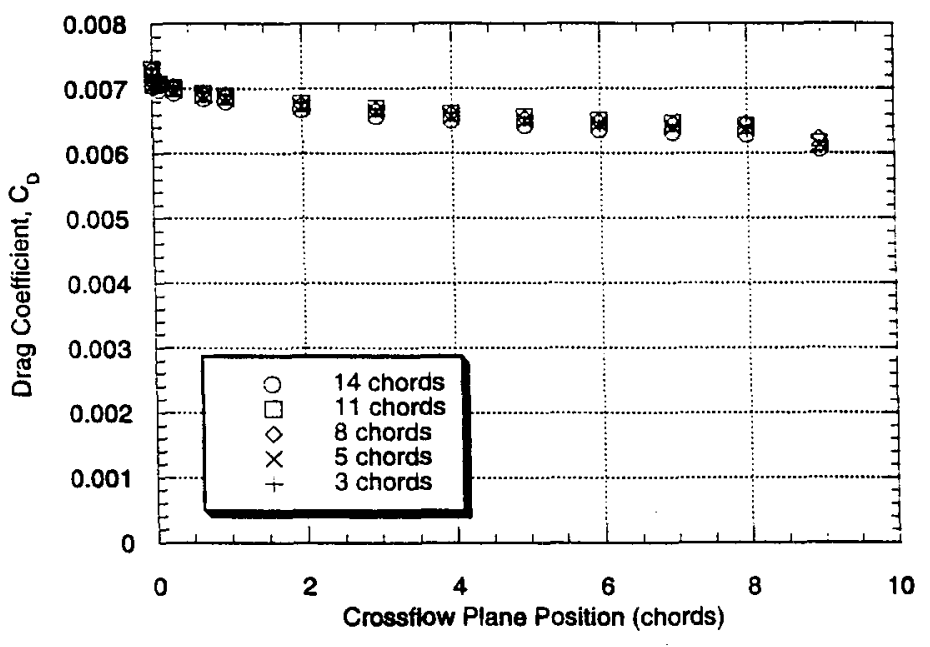

Fig. 2 Elliptic Wing-Effect of Bounding Box Size on Vortex Drag Coefficient. 


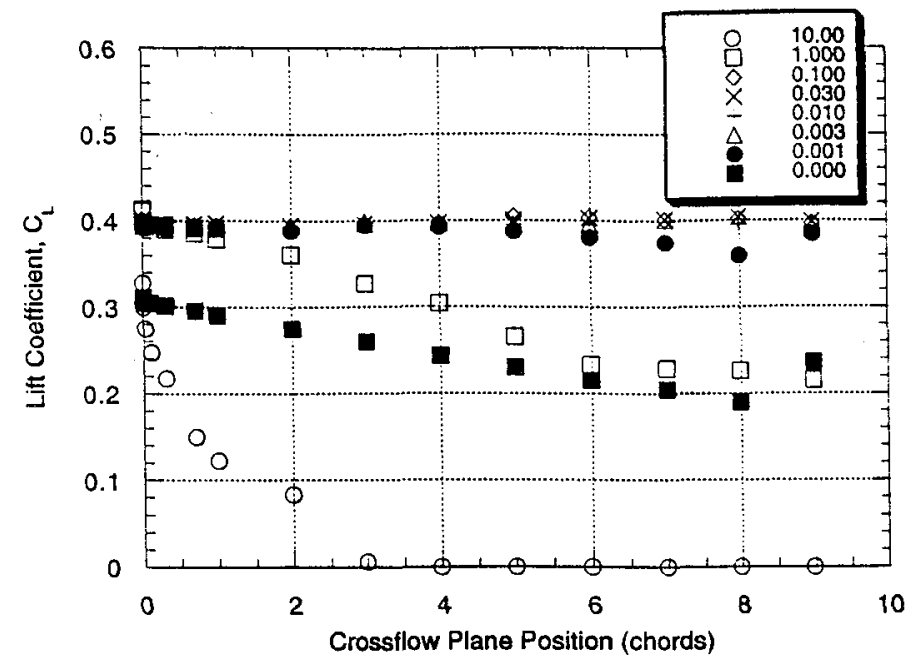

Fig. 3 Elliptic Wing-Effect of Level 1 Cutoff Parameter on Lift Coefficient.

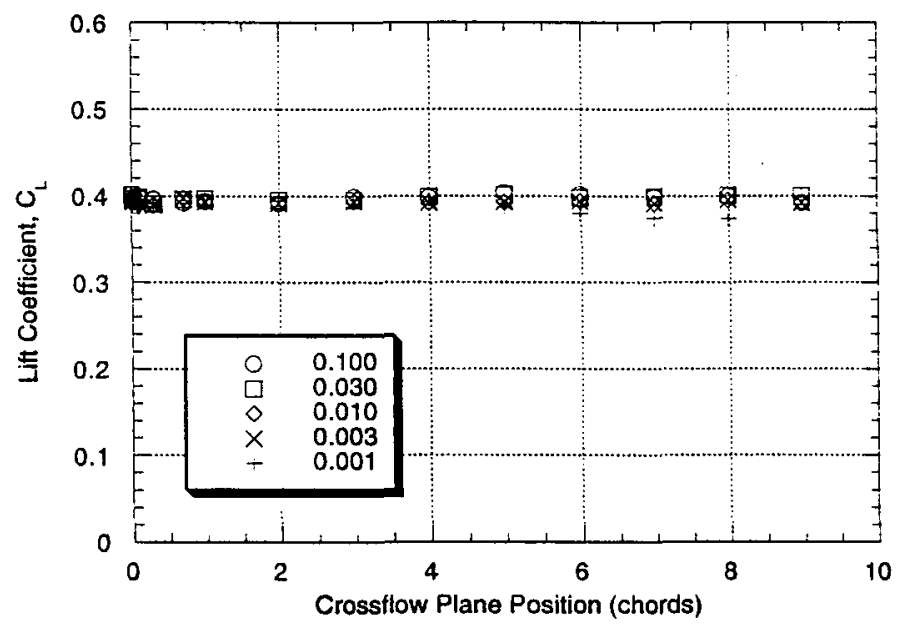

Fig. 5 Elliptic Wing-Effect of Level 1 Cutoff Parameter on Lift Coefficient; $C_{\zeta_{2}}=0.1$.

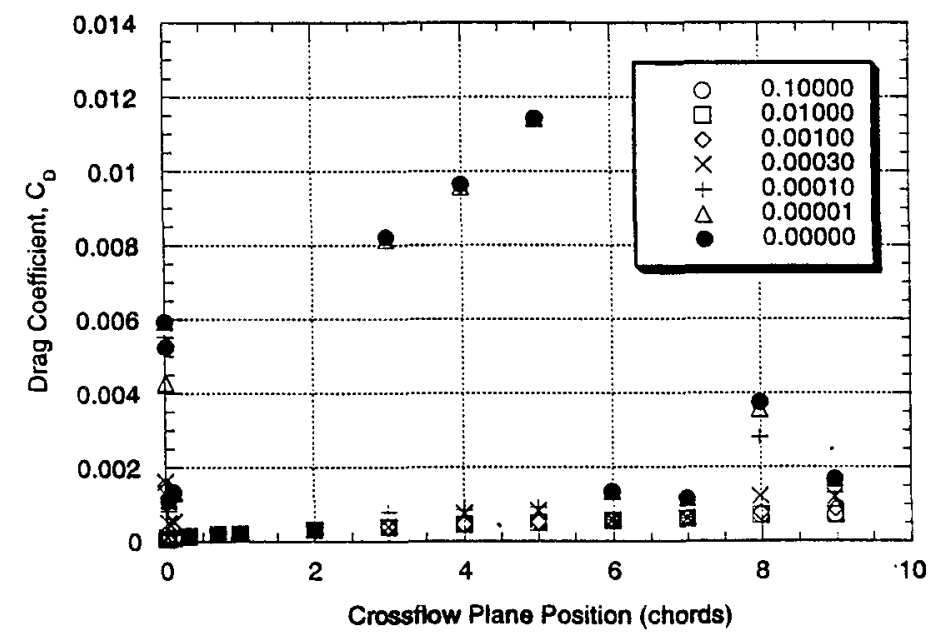

Fig. 7 Elliptic Wing-Effect of Level 1 Cutoff Parameter on Entropy Drag Coefficient; $C_{\zeta_{2}}=0.1$.

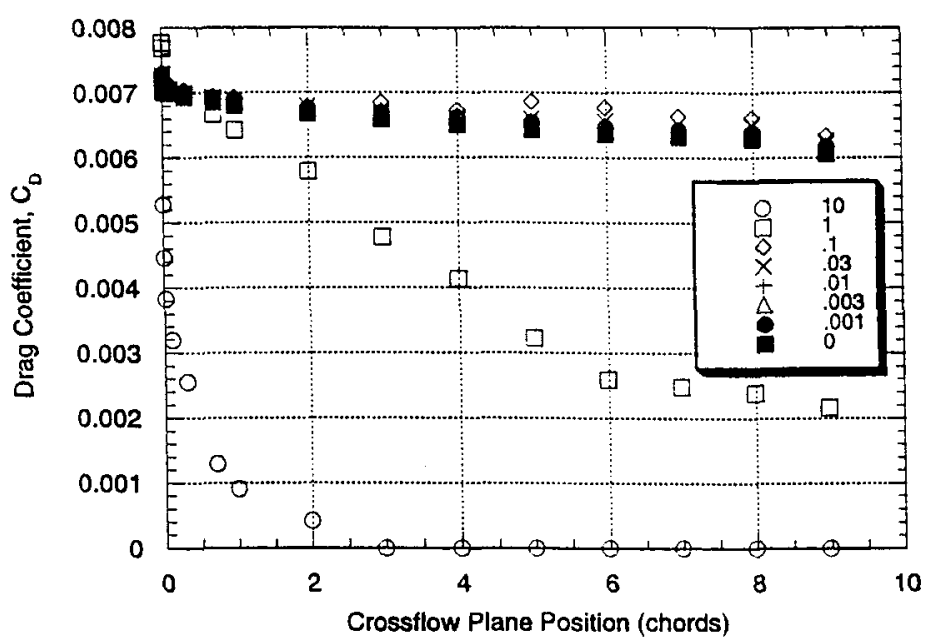

Fig. 4 Elliptic Wing-Effect of Level 1 Cutoff Parameter on Vortex Drag Coefficient.

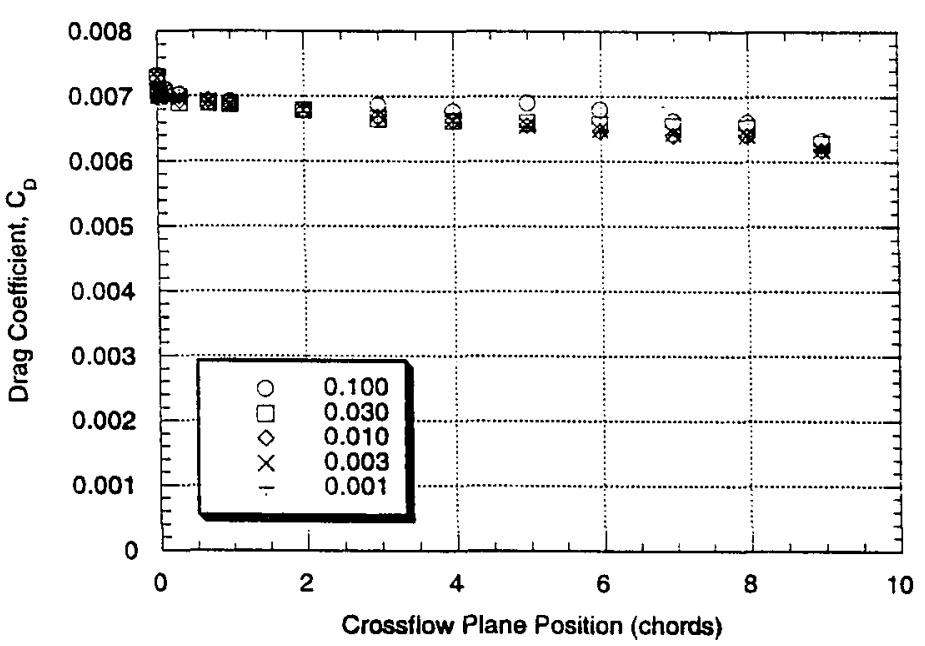

Fig. 6 Elliptic Wing-Effect of Level 1 Cutoff Parameter on Vortex Drag Coefficient; $C_{\zeta_{2}}=0.1$.

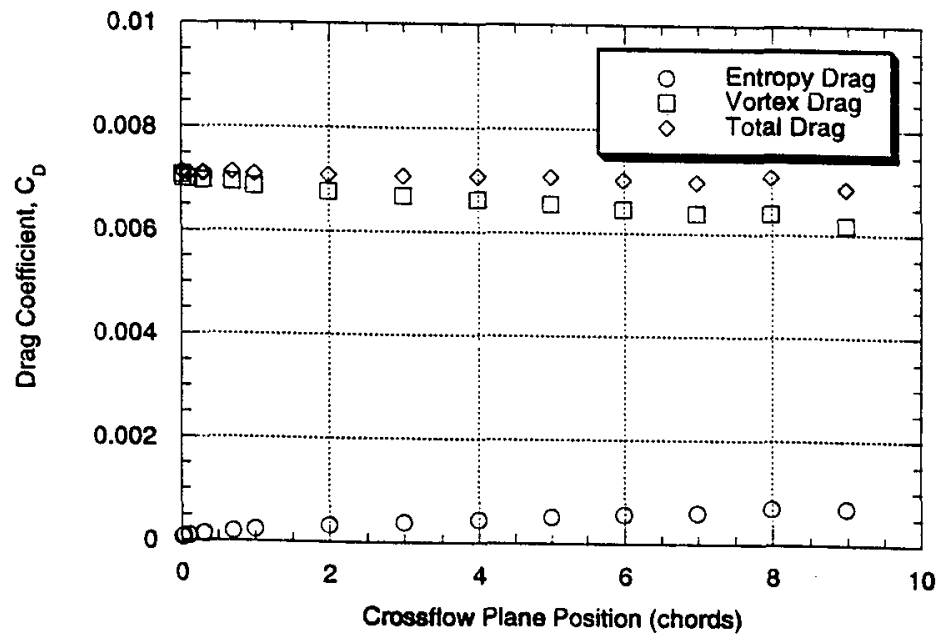

Fig. 8 Elliptic Wing-Total Drag Coefficient; $C_{\zeta_{1}}=0.01, C_{\zeta_{2}}=0.1, C_{s_{1}}=0.003$, and $C_{s_{2}}=0.1$. 


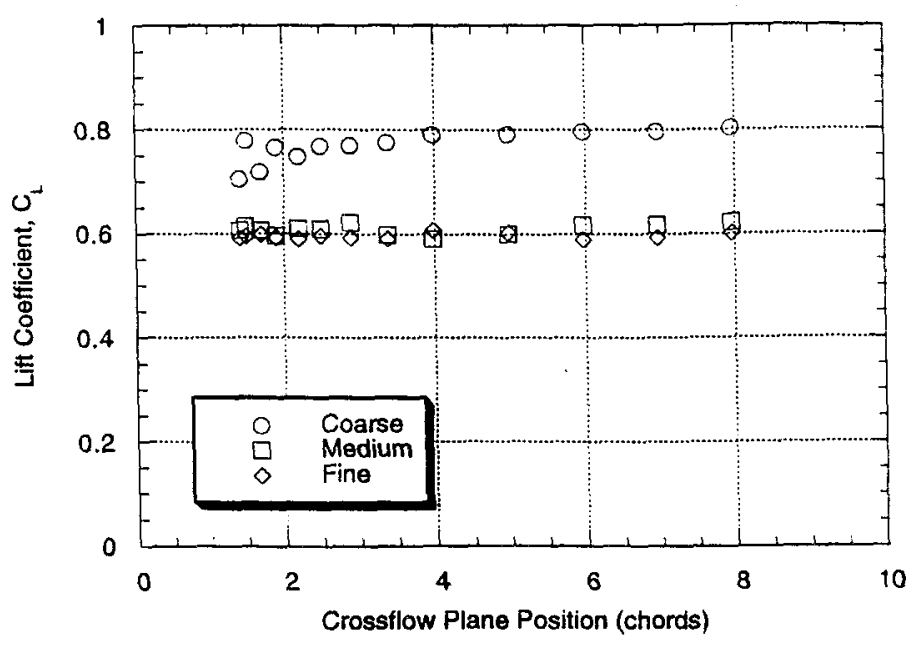

Fig. 9 M6 Wing-Effect of Grid Density on Lift Coefficient; Structured Grid.

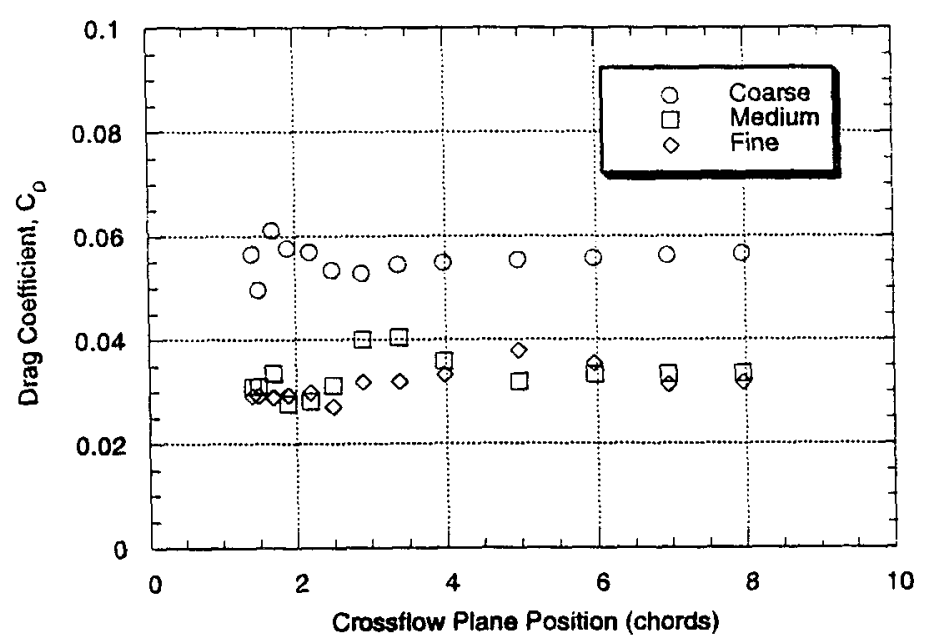

Fig. 11 M6 Wing-Effect of Grid Density on Entropy Drag Coefficient; Structured Grid.

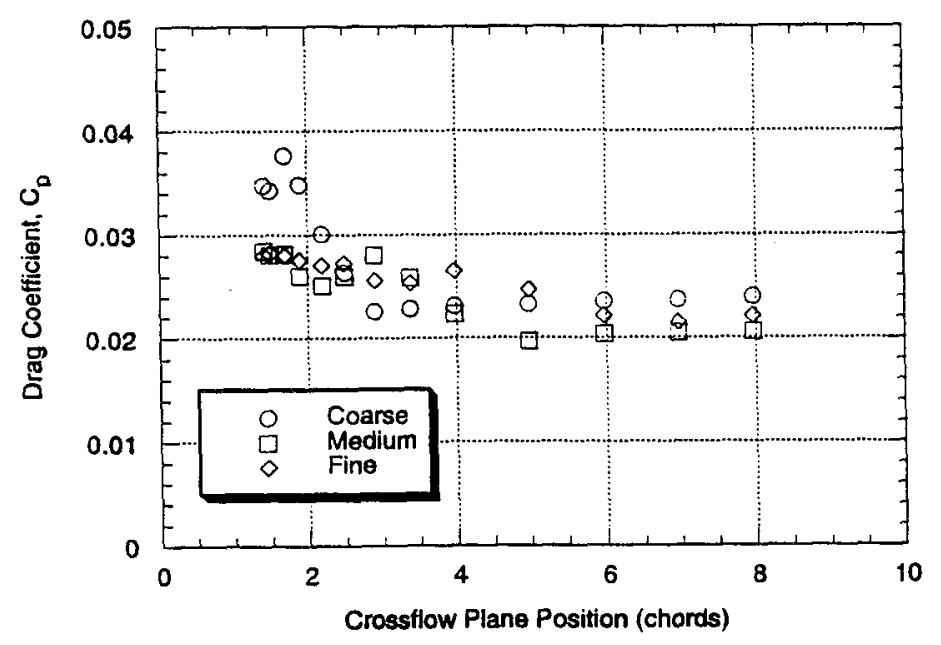

Fig. 13 M6 Wing-Effect of Grid Density on Vortex Drag Coefficient; Structured Grid.

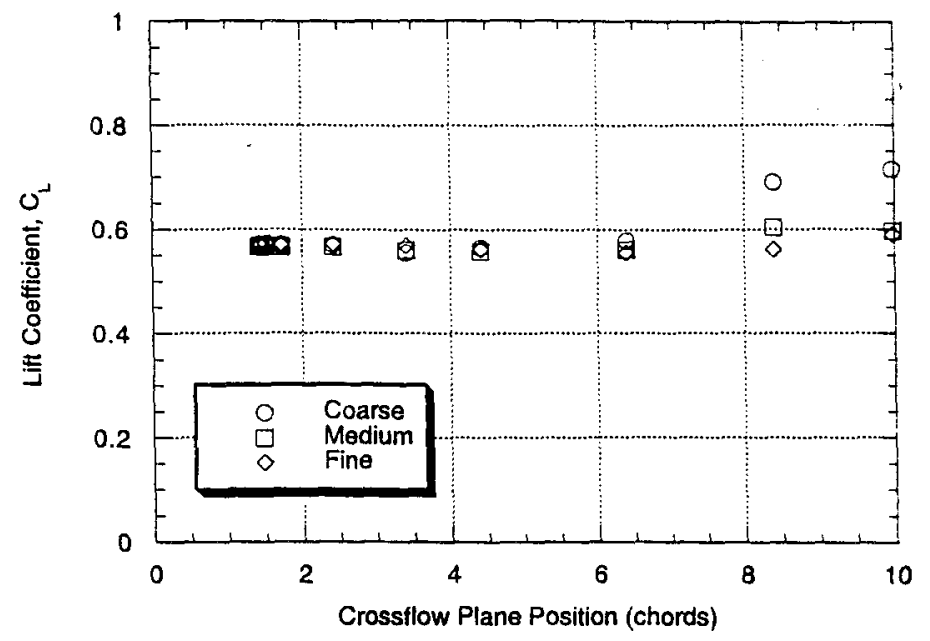

Fig. 10 M6 Wing-Effect of Grid Density on Lift Coefficient; Unstructured Grid.

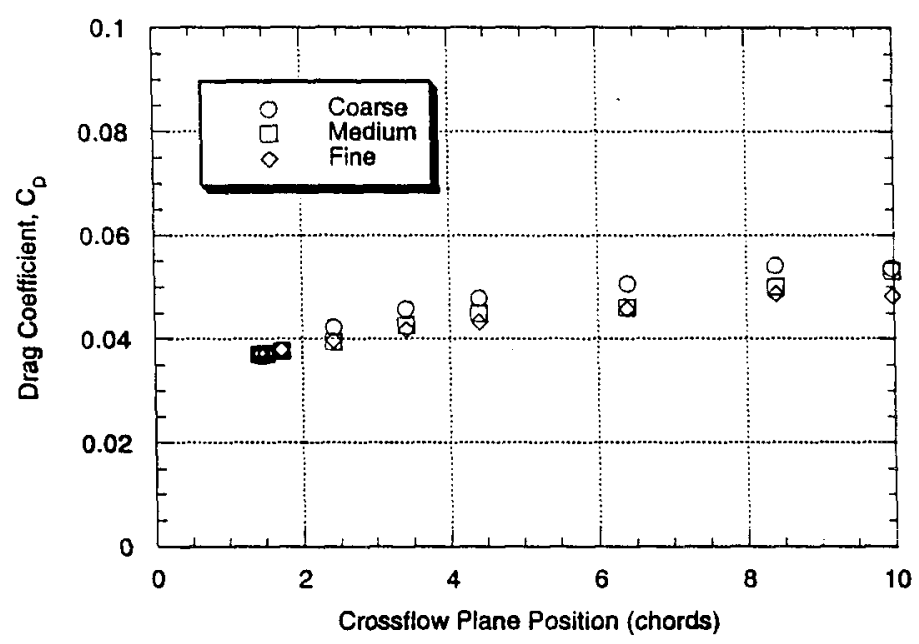

Fig. 12 M6 Wing-Effect of Grid Density on Entropy Drag Coefficient; Unstructured Grid.

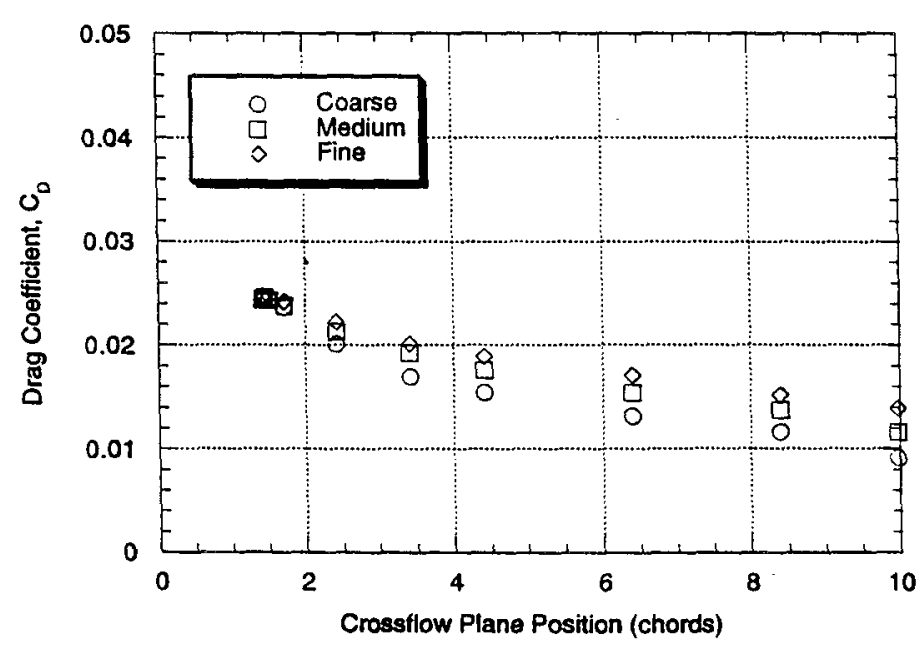

Fig. 14 M6 Wing-Effect of Grid Density on Vortex Drag Coefficient; Unstructured Grid. 


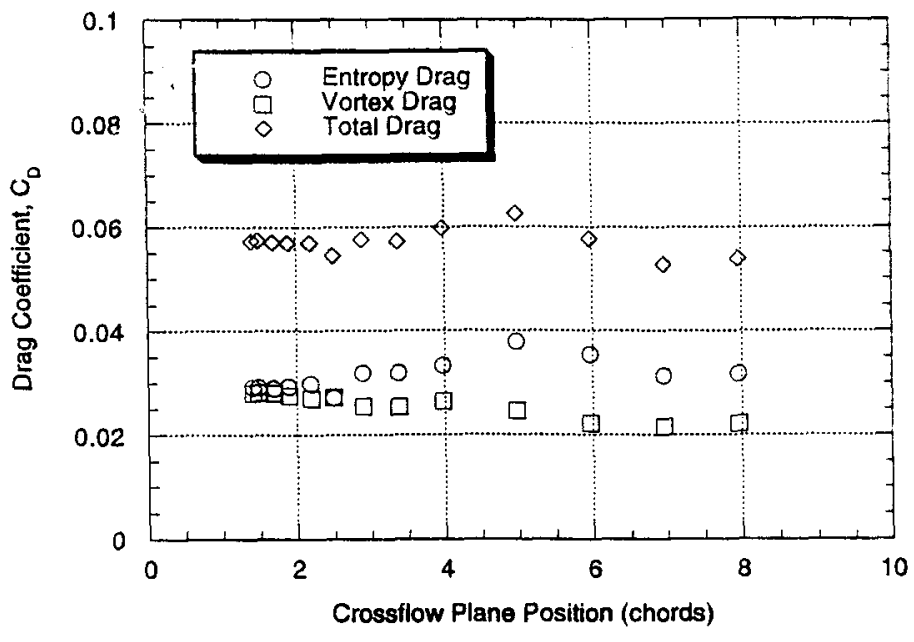

Fig. 15 M6 Wing-Total Drag Coefficient;

Structured Grid; $C_{\zeta_{1}}=0.01, C_{\zeta_{2}}=0.1$,

$C_{s_{1}}=0.003$, and $C_{s_{2}}=0.1$.

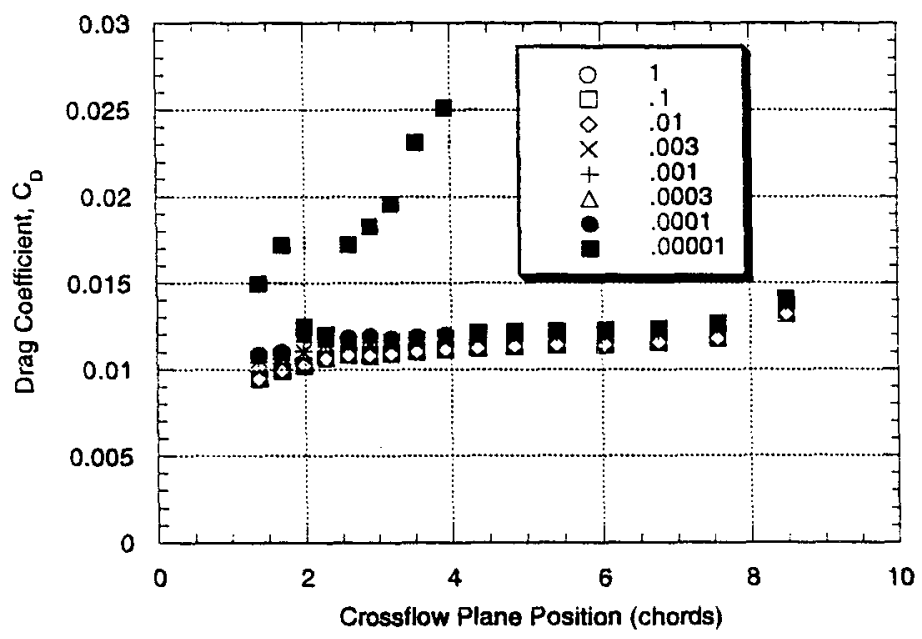

Fig. 17 W4 Wing-Body-Effect of Level 1 Cutoff Parameter on Entropy Drag Coefficient; $C_{\zeta_{2}}=0.1$.

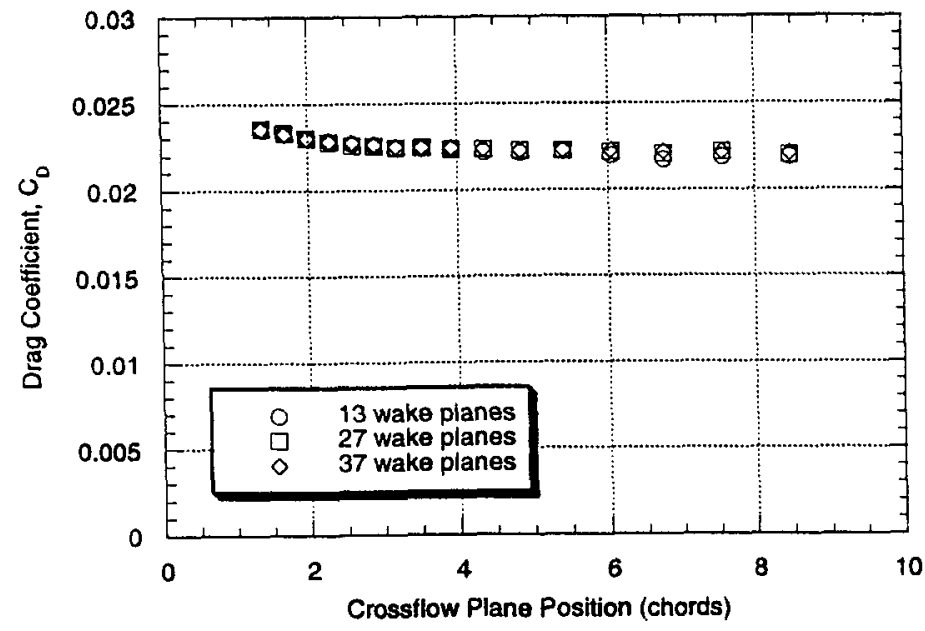

Fig. 19 W4 Wing-Body-Effect of Wake Grid Density on Vortex Drag Coefficient; $C_{\zeta_{1}}=0.01$, $C_{\zeta_{2}}=0.1, C_{s_{1}}=0.003$, and $C_{s_{2}}=0.1$.

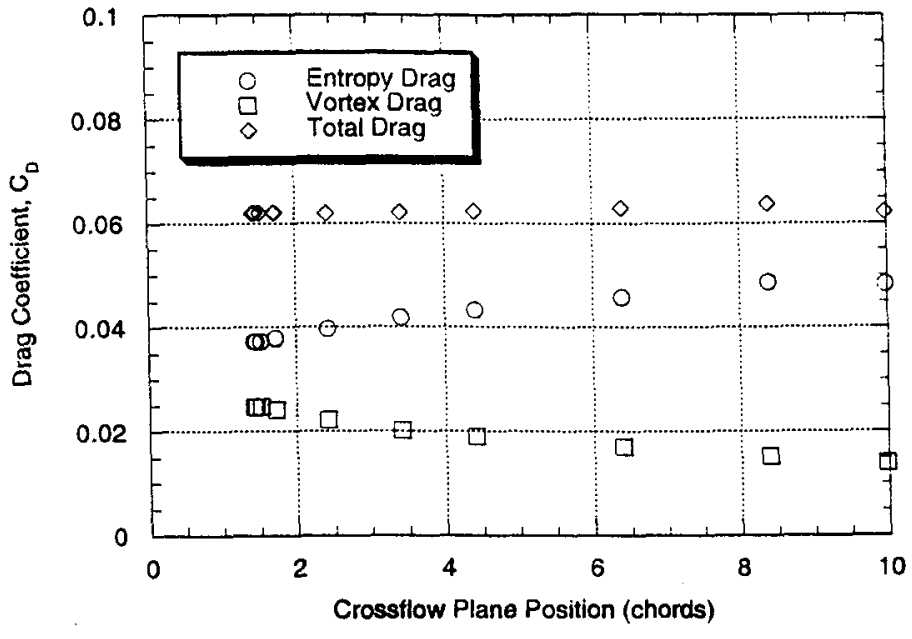

Fig. 16 M6 Wing-Total Drag Coefficient;

Unstructured Grid; $C_{\zeta_{1}}=0.01, C_{\zeta_{2}}=0.1$,

$C_{s_{1}}=0.003$, and $C_{s_{2}}=0.1$

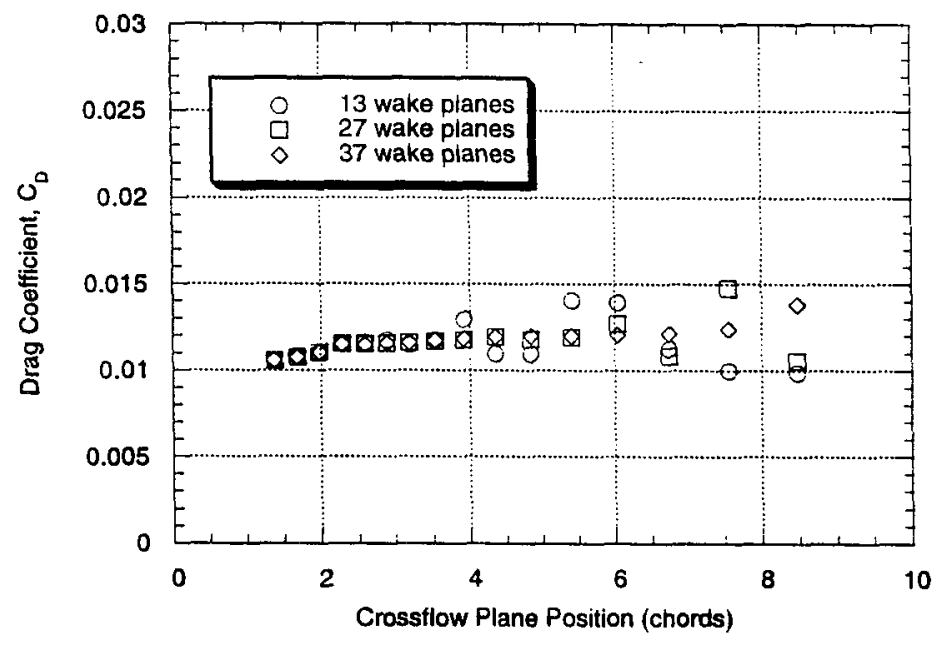

Fig. 18 W4 Wing-Body-Effect of Wake Grid Density on Entropy Drag Coefficient; $C_{\zeta_{1}}=0.01$, $C_{\zeta_{2}}=0.1, C_{s_{1}}=0.003$, and $C_{s_{2}}=0.1$.

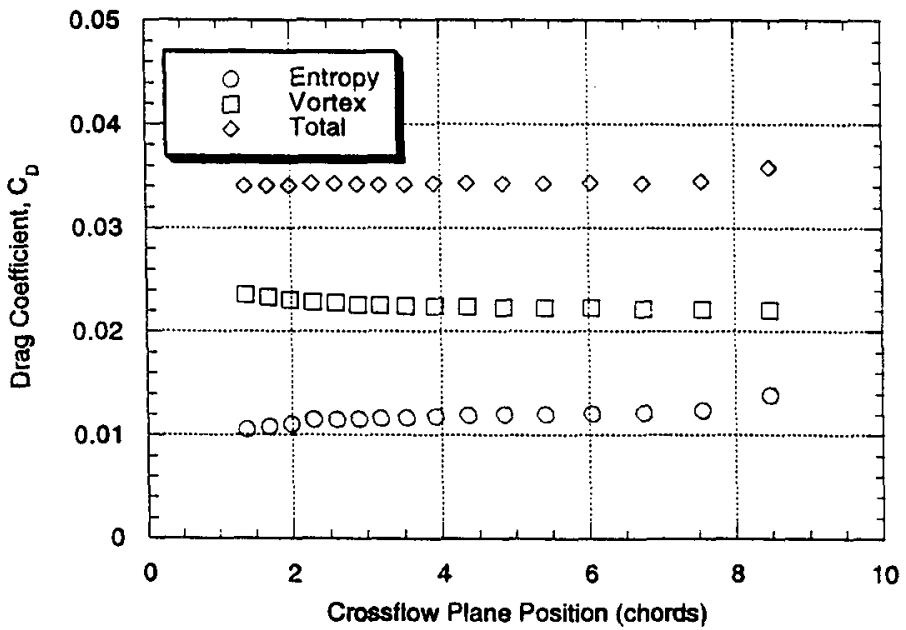

Fig. 20 W4 Wing-Body-Total Drag Coefficient; $C_{\zeta_{1}}=0.01, C_{\zeta_{2}}=0.1, C_{s_{1}}=0.003$, and $C_{s_{2}}=0.1$. 


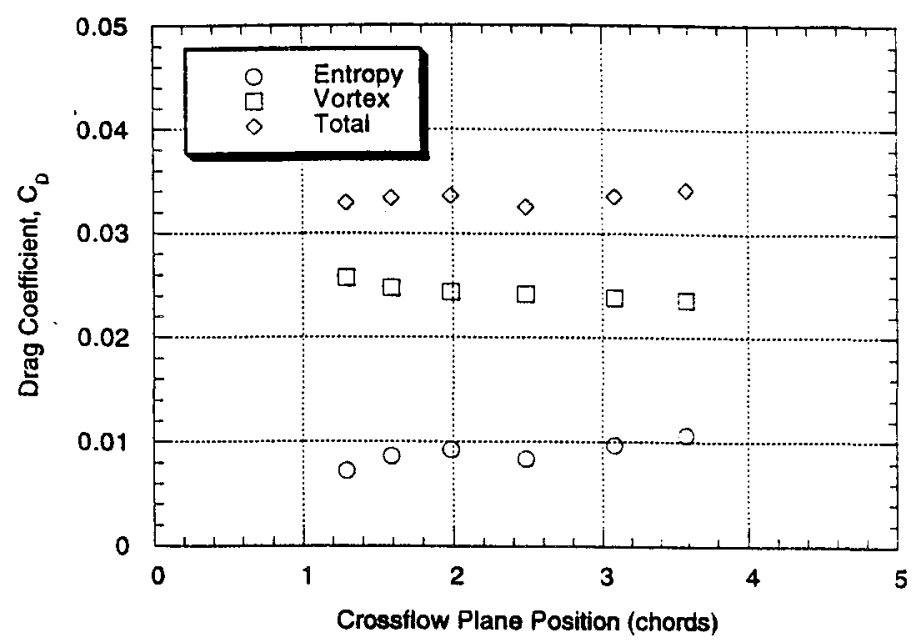

Fig. 21 M165 Canard-Wing-Total Drag

Coefficient; $C_{\zeta_{1}}=0.01, C_{\zeta_{2}}=0.1, C_{s_{1}}=0.003$, and $C_{s_{2}}=0.1$.

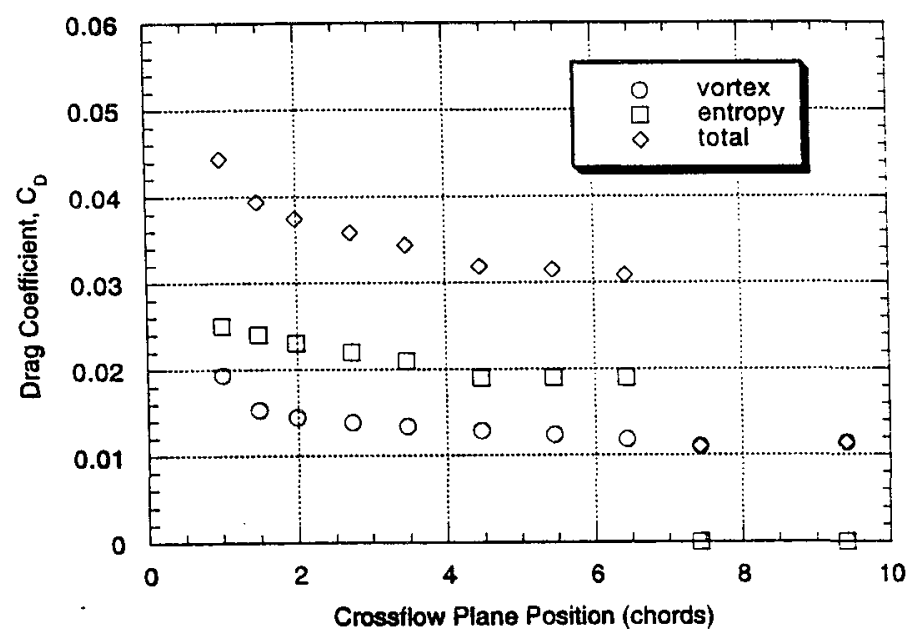

Fig. 22 NACA 0016 Wing-Total Drag

Coefficient; $C_{\zeta_{1}}=0.01, C_{\zeta_{2}}=0.1, C_{s_{1}}=0.003$, and $C_{s_{2}}=0.1$.

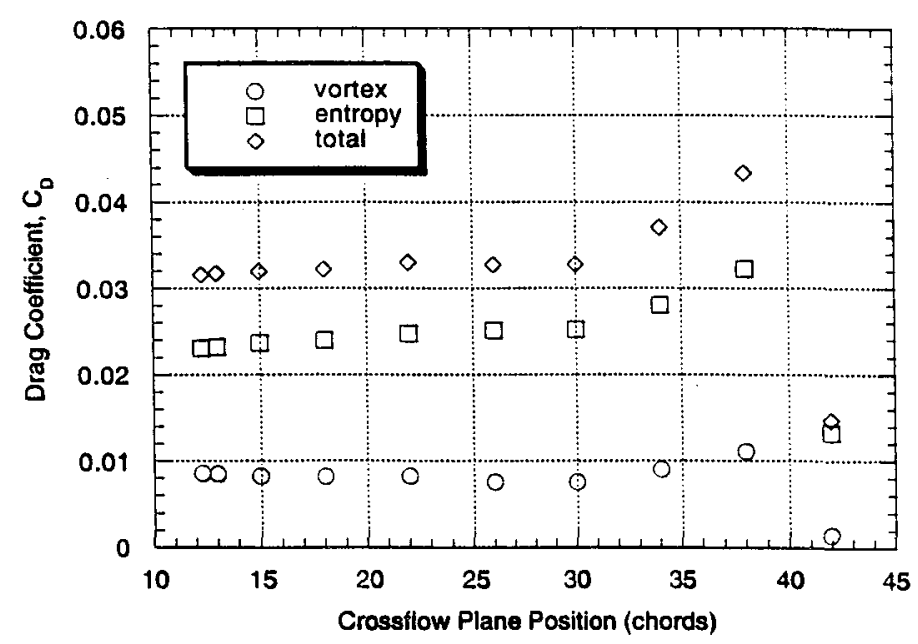

Fig. 23 Lockheed Wing A-Total Drag

Coefficient; $C_{\zeta_{1}}=0.01, C_{\zeta_{2}}=0.1, C_{s_{1}}=0.003$, and $C_{s_{2}}=0.1$. 